\title{
Experimental Study of Problem of Estrangement of AR. Drone Four Rotor Helicopter Velocity Parameter Calculated by the Internal IMU
}

\author{
Hideki Toda
}

Department of Electric and Electronic Engineering, University of Toyama, 3190 Gofuku, Toyama, 930-8555, Japan

\begin{abstract}
For four rotor helicopter system, the velocity parameter is important for stable real-time control, and the velocity is calculated by the IMU (Internal Measurement Unit) from the three-axis acceleration and gyro sensors.

Since the IMU unit developed and designed for multi-purpose use, high-frequency range of the acceleration/gyro sensors information was used for estimating the velocity and it is afraid to do overestimation of the velocity especially when the drone collision against the wall (even with a slight contact) and the impact due to blasts. The result shows that the IMU unit calculated velocity was divergent 20 times from the real velocity measured from the camera on the ceiling even when low speed contact with a wall $(25 \mathrm{~cm} / \mathrm{sec}$ speed). When the estimated velocity parameter would be used for the position control and stabilizing of AR. Drone helicopter, it will be necessary to consider the problem of the estrangement of the velocity to develop the drone system for inspection at infrastructure equipment such as the bridge or tunnel.
\end{abstract}

Keywords—Four rotor helicopter, AR. Drone 1.0, Velocity estimation, IMU, Investigation of bridge or tunnels.

\section{INTRODUCTION}

In this paper, the velocity parameter obtained from the IMU (Internal Measurement Unit) in AR. Drone four rotor helicopter 1.0 was measured and analyzed, and it was found that the velocity parameter was calculated as an estrangement fashion when the drone touch against a wall with a slight impact. The IMU is one of the standard equipment of the drone system, since the information of acceleration and gyro sensors is indispensable for real-time stable posture controlling [1-7]. In addition, the velocity parameter is also estimating at the same time in the IMU, and it was typically used for the "space position" controlling and stabilizing process for drone controlling [1,8-16]. Since the IMU is developed and designed for multi-purpose use, high-frequency range of the acceleration and gyro sensors information was used for estimating the velocity parameter and it is afraid to do overestimation of the velocity especially when the drone collides against object such as wall or tree branches (Fig.1).Also, impacts from wind flow blasts would be afraid to do overestimation of the velocity. The main object of this paper is to compare the real velocity value measured from the camera on the ceiling with the IMU output estimated velocity parameter in the $x-y$ space in the room (Fig.2) to confirm the effect of the overestimation of the velocity parameter by the IMU.

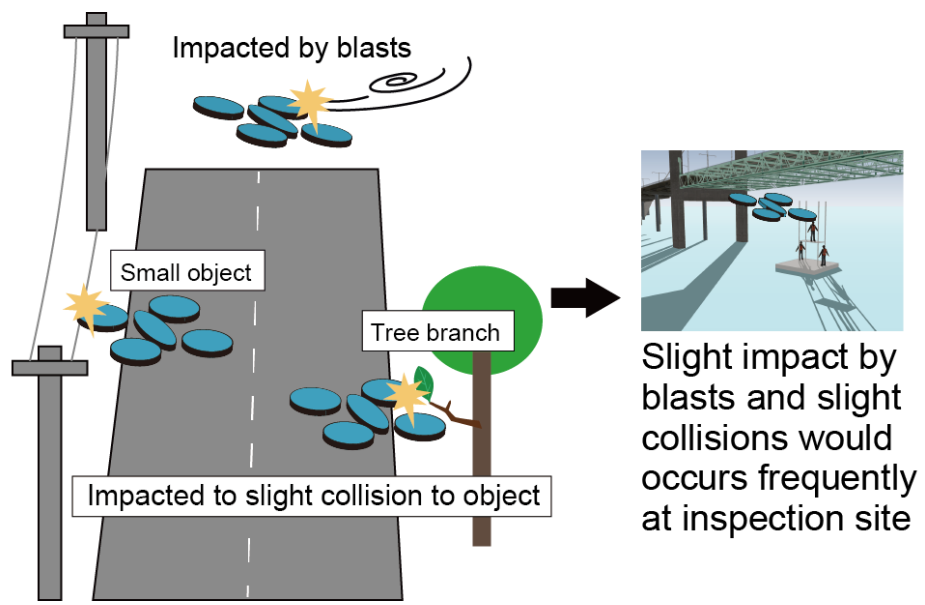

FIG.1. IMPACTED BY BLASTS AND SLIGHT COLLISION TO OBJECT WOULD OCCURS FREQUENTLY WHILE INSPECTING BRIDGE OR TUNNEL BY USING A DRONE SYSTEM.

Twenty percent of currently 730,000 bridges are under construction for more than 50 years in Japan [8].Many business operations of periodic inspection of the under bridge, inside tunnels and buildings are beginning to be tried [17-19]. Even if 
the drone would be controlled by a human being, the flight by visual confirmation of man is difficult in the over $10 \mathrm{~m}$ long distance flight $[2,3,8-10,20-23]$.In many cases, the periodic inspection of a bridge is necessary to operate in a place $200 \mathrm{~m}$ away, and it is impossible to remove the influence of contact and blasts around the four rotor machine that cannot be visually observed $[1,14,17,18]$.

\section{PREVIOUS STUDY AND THE MATHEMATICAL BACKGROUND}

Four rotor helicopter system would not be included autonomous position controlling way as itself, and the positioning control is necessary for the space using InfraRed 3D cameras [3,12] or GPS sensor system [14, 18] to control the drone position [3, 13-15, 24-27].In the InfraRed 3D camera, the precision of the position measurement is $1 \mathrm{~mm}$ order. However, the area of the using this method is within $10 \mathrm{~m}$ and the indoor situation only [2]. On the other hand, if the drone would be controlled in the outdoor situation, the GPS signal can be used that there is no obstacles upper direction (sky) and movement direction. However, to use the drone for the periodic inspection of under the bridge or inside tunnels, the two approaches cannot be adopted since the instability of the GPS signal under these sites [17]. Also, in such situations of the long distance periodic inspection (such a case $200 \mathrm{~m}$ distance from the base), camera image feedback control (or by visual confirmation of man from the base point) is necessary.

To realize stable flight control at the long-distance situation (over $10 \mathrm{~m}$ ), the camera image feedback control would become important, on the other hand, autonomous flight stabilizing process using the IMU information would be more indispensable since slight touching to thin tree branches and impacts from wind flow blasts could not correctly confirm from the base point visually [3].To suppress the deviation of trajectory due to the disturbances such as wind flow, one of the main factors of the feedback control is velocity parameter $\vec{v}$. Equation of motion of the drone while floating was described as (not included four rotors depend on complex dynamics, simple one-mass equation of motion).

$$
m \vec{a}=\vec{F}=-k \vec{v}+\vec{f}
$$

where $m$ is a mass, $\vec{a}$ is the acceleration, $\vec{v}$ is the velocity, $k$ is friction coefficient by air and $\vec{f}$ is the external control input. In Eq.1, the term $-k \vec{v}$ has movement vibration suppression influence as the friction, and it can stabilize the drone movement, but the effect is limited small since the $k$ is very small and unstable.

To realize the stabilization, the effect of the D (differential) gain is typically used as below,

$$
\vec{f}=-k_{d} \overrightarrow{v^{e}}
$$

where $k_{d}$ is constant feedback differential gain parameter, $\vec{v}$ is estimated velocity calculated by the IMU. Generally, the real velocity $\vec{v}$ and the estimated velocity $\overrightarrow{v^{e}}$ (calculated value by the IMU) take same value under normal flight situation, and the term $-k_{d} \overrightarrow{v^{e}}$ affects the movement vibration suppression. The effect is typically large comparing with the air friction $-k$.

On the other hand, to calculate the estimated velocity $\overrightarrow{v^{e}}$, the IMU have to estimate $\overrightarrow{v^{e}}$ by using two sensor outputs - the internal acceleration and gyro sensor. Since the IMU is developed and designed for multi-purpose use, high-frequency range of the acceleration and gyro sensors information is used for precise estimating of $\overrightarrow{v^{e}}$. Especially, the high-frequency (few hundred hertz) information is indispensable to estimate it. But when the drone would collide with some slight touching to thin tree branches or impacts from blasts in the outdoor inspection works, there is a possibility that an unpredictable highfrequency change of the acceleration and gyro sensors would distort the $\overrightarrow{v^{e}}$. As a result, it is afraid to shift the velocity value especially when the drone collides against some object or would be impacted by blasts.

\section{METHOD}

In this experiment, AR. Drone 1.0 quad rotor model helicopter developed by Parrot Co. [28] was used. In Fig. 2, the aircraft is positioned at the center of the room $(5 \times 6 \mathrm{~m}$ square room, height is $2.4 \mathrm{~m})$ and it flows automatically with the height of 0.6 $\mathrm{m}$ from the ground. To control the movement of the aircraft, AR. Drone library for Processing named ARDroneForP5 as developed by Y.Shigeo [28] was used and it was connected to a PC with Wi-Fi network. The basic period of movement command transmission between the aircraft and the PC was about $99.6 \mathrm{msec}$ (18.4 msec standard deviation S.D.). The estimated velocity $\overrightarrow{v^{e}}$ can be measured from the internal IMU of the drone via the control library ARDroneForP5 with about $10 \mathrm{~Hz}$ speed. 


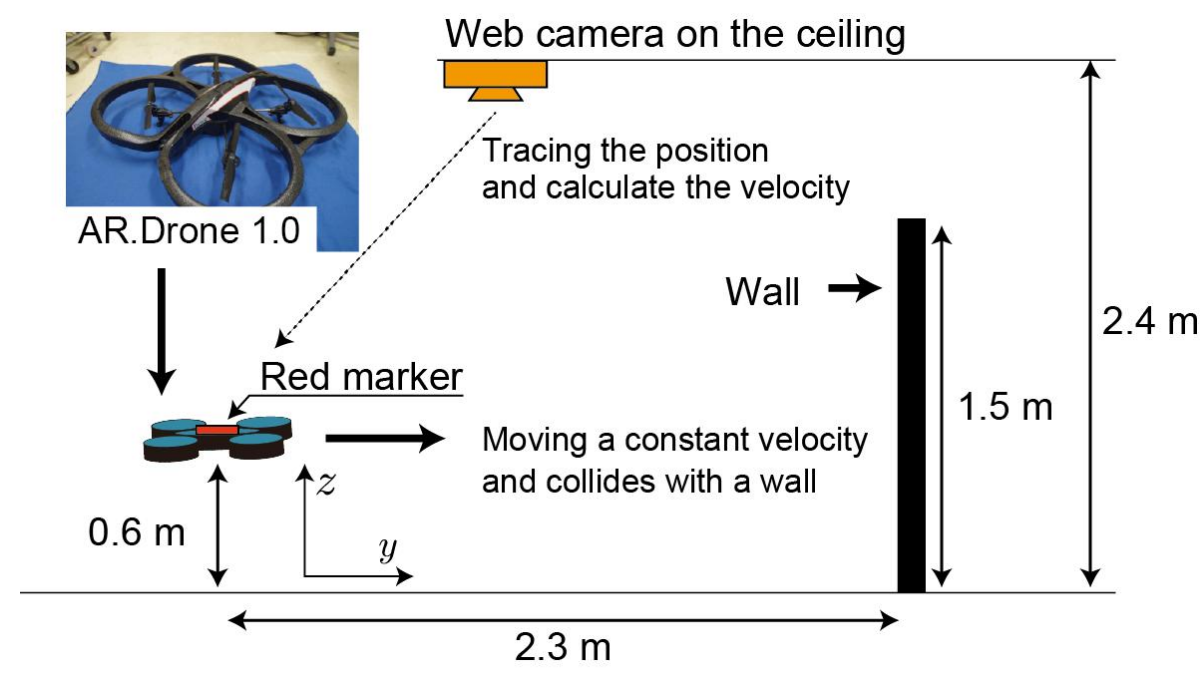

\section{FIG.2. SIDE VIEW OF THE EXPERIMENTAL SETUP. AR.DRONE WAS CONTROLLED TO A CONSTANT VELOCITY MOVEMENT, AND COLLIDED WITH A WALL AT THE CONSTANT VELOCITY. THE REAL DRONE MOVEMENT VELOCITY WAS MEASURED BY A WEB CAMERA AT THE CEILING.}

Fig. 3 shows a top view of the experimental setup. The real drone position in the $x-y$ space (Fig. 3 ) was measured by a web camera (BSW20KM11, 640×480, $60 \mathrm{fps}, 130 \mathrm{deg}$ visual angle, iBUFFALO Co.) attached to the ceiling of the room. The position discrimination was about $2 \mathrm{~mm}$. To reduce measurement noise of the real velocity, a low pass filter was applied to the real velocity value calculated from the position data obtained at $60 \mathrm{~Hz}$. Time constant was defined about $100 \mathrm{msec}(10$ $\mathrm{Hz}$ ).From the result of the drone position, the real velocity $\vec{v}$ can be calculated.

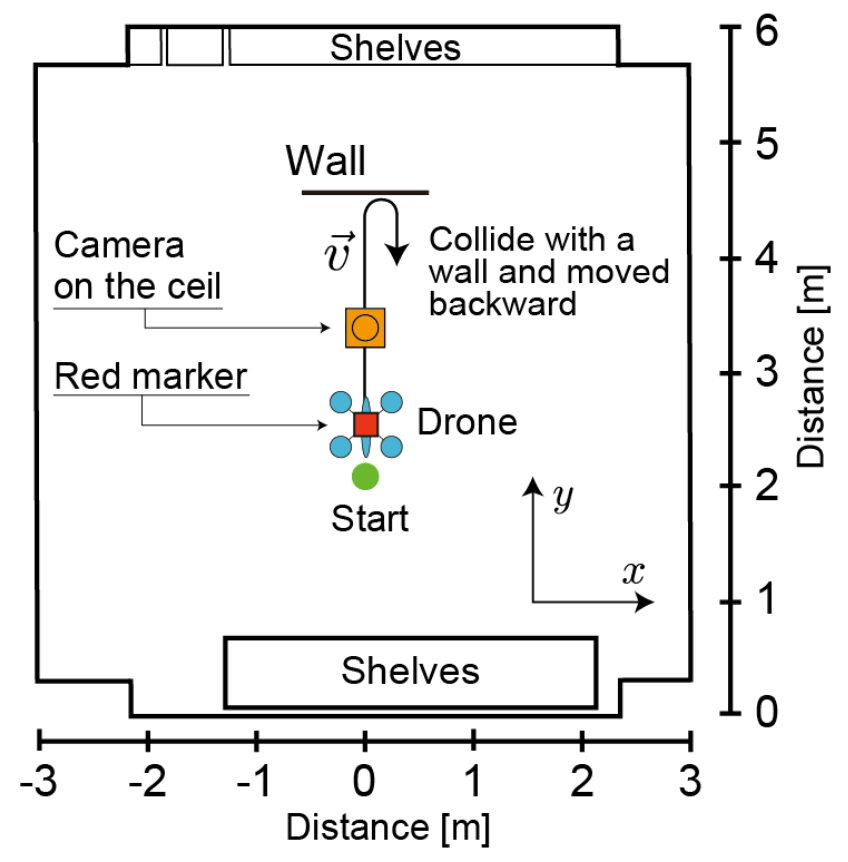

FIG.3. SETUP OF AR. DRONE COLLIDING EXPERIMENT TO A WALL WITH A CONSTANT VELOCITY $\overrightarrow{\mathbf{v}}$ (TOP VIEW).

To compare the two velocity values while the collision, $y$ axis values of the velocity $\vec{v}$ and $\overrightarrow{v^{e}}$ were used in Fig.3. It were denoted as $v_{y}$ and $v_{y}^{e}$ respectively. The drone's movement roll and pitch speed commands $V_{\text {command }}^{r}=x$ axis, $V_{\text {command }}^{p}=y$ axis and the yaw (spin) control command $V_{\text {command }}^{s}$ are determined.

$$
\left\{\begin{array}{l}
V_{\text {command }}^{r}=0 \\
V_{\text {command }}^{p}=\gamma_{p} \\
V_{\text {command }}^{s}=0
\end{array}\right.
$$


Where $\gamma_{p}$ is constant value $[2,10,20-22]$.

\section{EXPERIMENT}

There are two phases of the movement control in the experiment. (1) Take off and 5 sec fixed point floating phase: The $\gamma_{p}$ set as 0 before the drone take off. After the take-off, $5 \mathrm{sec}$ normal flight (without any control of the drone) is implemented to stabilize the posture. Since the phase (1) was totally within $10 \mathrm{sec}$, there is slight yaw change $(<0.5 \mathrm{deg})$ and slight movement $(<15 \mathrm{~cm})$ by the drone implemented posture control system. From the reason, we do not send any control commands to the drone while phase (1).

(2) Constant velocity and backward (slowdown) control phase: Firstly, the $\gamma_{p}$ set as 30 or 100 (max 100 in the ARDroneForP5 library) as constant velocity movement ( $+y$ axis direction) after the phase (1). Next, when the drone is crossed the position of the camera on the ceiling, the constant velocity parameter $\gamma_{p}$ is changed to -100 , and the drone was tried to backward movement ( $-y$ axis direction) with the inertial motion continued. Since the positive velocity $v_{y}$ of the drone is continuously reducing while the backward control phase, the $v_{y}$ would pass through 0 and take negative value. In this phase (2), the drone would collide with the wall at a collision velocity, and the collision velocity $v_{y}^{c}$ mainly depends on the firstly set $\gamma_{p}$ value (30 or $100 \%)$.

\section{RESULT}

Figure 4 represents the result of comparison between the real velocity $v_{y}$ of $y$ axis in Fig. 3 and the estimated velocity $v_{y}^{e}$ of $y$ axis obtained from the AR.Drone's IMU. Horizontal axis means elapsed time $t$ [sec].In this experiment, the standard deviation (S.D.) of the real position of $x$ was measured as $6.7 \mathrm{~cm}$ while $2.3 \mathrm{~m}$ flight, and it means that there was little movement of $x$ axis while the movement. Filled Square mark means the real velocity $v_{y}$ measured from the camera on the ceiling, and the white square mark is the estimated velocity $v_{y}^{e}$ obtained from the AR.Drone's IMU in each time period [sec]. Two velocity transitions vary in coincidence, and the deviation (S.D. of $v_{y}-v_{y}^{e}$ ) was calculated as $23.4 \mathrm{~cm} / \mathrm{sec}$.

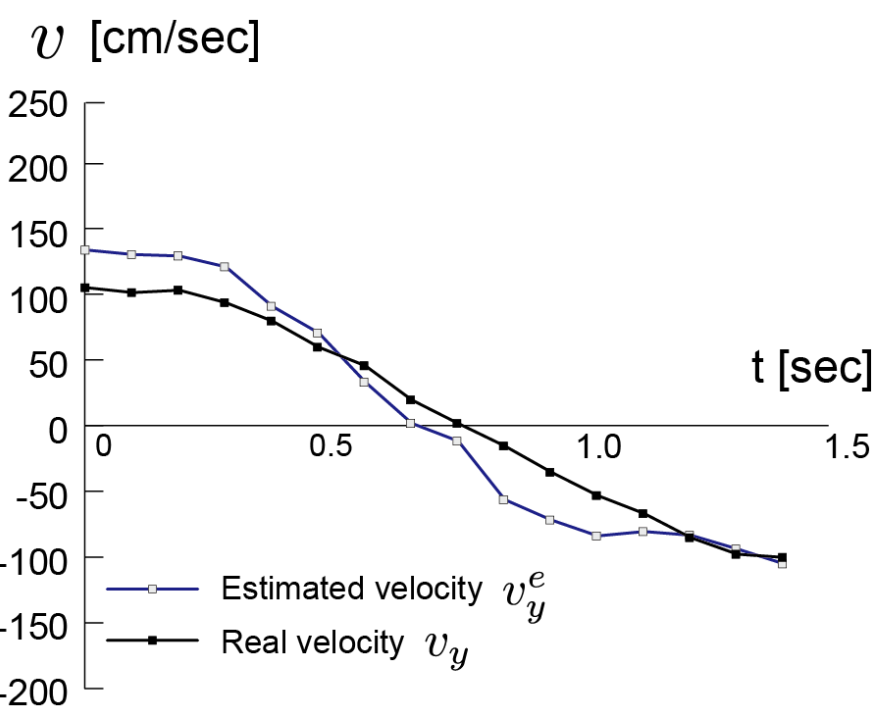

\section{FIG.4. COMPARISON BETWEEN THE REAL VELOCITY $v_{\mathrm{y}}$ OF $\mathrm{y}$ AXIS AND THE ESTIMATED VELOCITY vy $\mathrm{y}_{\mathrm{e}}^{\mathrm{e}}$ TAINED FROM THE AR.DRONE'S IMU. THERE IS NO COLLISION CASE WITH THE WALL. HORIZONTAL AXIS REPRESENTS TIME [sec]}

Figure 5 represents the result of comparison between the real velocity $v_{y}$ of $y$ axis and the estimated velocity $v_{y}^{e}$ of $y$ axis obtained from the AR.Drone's IMU when there is a slight collision with a wall. Even in this experiment, the two velocity transitions vary in coincidence, but in the timing of vertical dotted line (collision timing), the estimated velocity $v_{y}^{e}$ suddenly transitioned to a high positive value $(205 \mathrm{~cm} / \mathrm{sec})$ and it was decreasing and approaching to real velocity value $v_{y}$ with a large delay. Immediately after the collision, the real velocity was $v_{y}=-10.0 \mathrm{~cm} / \mathrm{sec}$.In the timing of the collision, the real velocity $v_{y}$ takes about $25 \mathrm{~cm} / \mathrm{sec}$, and this velocity estrangement phenomenon was observed in almost all collisions. There is an over 20 times difference between the $v_{y}^{e}$ and $v_{y}$ when the collisions $\left(v_{y}^{e} / v_{y}=\frac{205 \mathrm{~cm} / \mathrm{sec}}{10.0 \mathrm{~cm} / \mathrm{sec}}=20.5\right)$. 


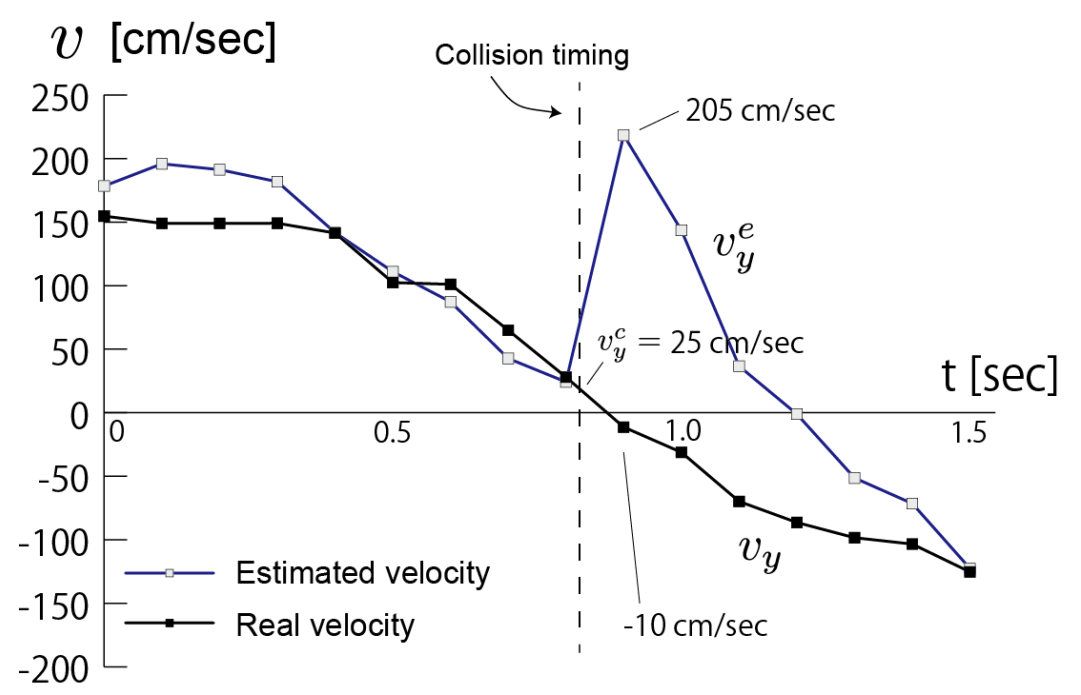

\section{FIG.5. COMPARISON BETWEEN THE REAL VELOCITY $v_{\mathbf{y}}$ OF y AXIS AND THE ESTIMATED VELOCITY $v_{y}^{\mathrm{e}}$ OBTAINED FROM THE AR.DRONE'S IMU WHEN THE DRONE COLLIDES WITH THE WALL SOFTLY (ABOUTV $=25 \mathrm{CM} / \mathrm{SEC})$}

Figure 6 represents the result of comparison between the real velocity $v_{y}$ and the estimated velocity $v_{y}^{e}$ when the drone was collided to a wall with a high velocity (about $v_{y}^{c}=60 \mathrm{~cm} / \mathrm{sec}$ ). The two velocity transitions vary in coincidence basically until the collision timing (vertical dotted line), the estimated velocity $v_{y}^{e}$ suddenly transitioned to a positive high value (maximum $425 \mathrm{~cm} / \mathrm{sec}$ ) and it was decreasing and approaching to real velocity value $v_{y}$ with a large delay. The real velocity was measured as $-5 \mathrm{~cm} / \mathrm{sec}$ in the above timing. In the timing of the collision, the real velocity $v_{y}$ takes about $60 \mathrm{~cm} / \mathrm{sec}$.

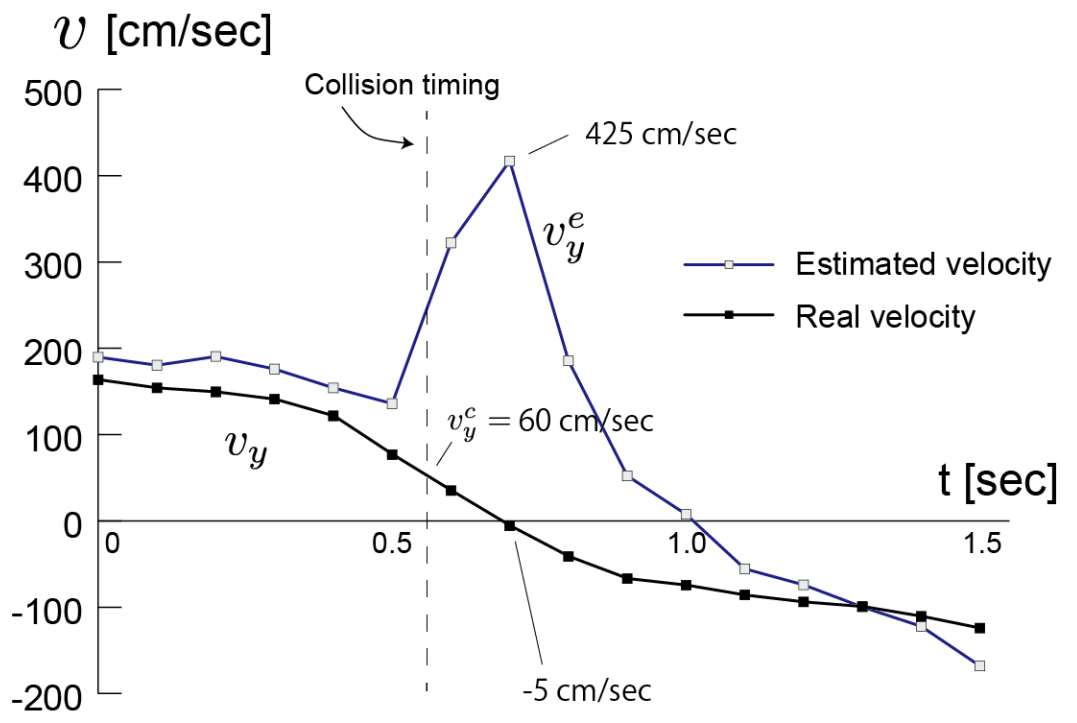

\section{FIG.6COMPARISON BETWEEN THE REAL VELOCITY $v_{y}$ OF $y$ AXIS AND THE ESTIMATED VELOCITY $v_{y}^{e}$ OBTAINED FROM THE AR.DRONE'S IMU WHEN THE DRONE COLLIDES WITH THE WALL HARDLY (ABOUTV $=60 \mathrm{CM} / \mathrm{SEC})$}

From above three experiments, the estimated velocity $v_{y}^{e}$ was estranged from the real velocity $v_{y}$ after the collision timing with the wall. Maybe multiple causes are considerable to explain the phenomenon, however, if the drone use the $v_{y}^{e}$ to stabilize the position movement that was discussed in section 2, the estrangement of the velocity would affect incorrect D gain feedback as denoted by Eq.2.

\section{DISCUSSION}

The reason for the estrangement of the estimation velocity $v_{y}^{e}$ would be induced by the velocity estimating algorithm implemented in the IMU. Since IMU is generally developed and designed for multi-purpose use, it would use high-frequency 
range of the acceleration and gyro sensors information to estimate the velocity, and there is a problem that the influence of high-frequency information affecting to the velocity estimation would be unstable especially when the IMU is impacted by small tree branches collision or blasts to four rotors system. If the IMU does not use the high-frequency information, the precision of the estimated velocity would be reduced, and imprecision in the velocity estimation would make control of the spatial position of the four rotors system inaccurate (see Eq.2). To improve the accuracy of the spatial position control, high precision velocity estimation is necessary, the two factors are in a trade-off relationship and it would be difficult to analyze this trade-off problem. The estrangement of the estimation velocity is necessary to take care when the subject uses the estimation velocity factor to stabilize the AR. Drone 1.0 helicopter at least.

\section{CONCLUSION}

In this paper, the velocity parameter obtained from the IMU (Internal Measurement Unit) in AR. Drone four rotor helicopter 1.0 was measured and analyzed, and it was found that the velocity parameter was calculated as an overestimated fashion when the drone touch against the wall with a slight impact. For four rotors helicopter system, the velocity parameter is important for stable real-time control, and the velocity is generally calculated by the IMU (Internal Measurement Unit) from the three-axis acceleration and gyro sensors. The result shows that the IMU estimating velocity $v_{y}^{e}$ was divergent 20 times from the real velocity $v_{y}$ measured from the camera on the ceiling even when low speed contact with a wall $(25 \mathrm{~cm} / \mathrm{sec}$ speed). When the estimated velocity parameter would be used for the position control and stabilizing of AR. Drone helicopter, it will be necessary to consider the problem of the estrangement of the velocity to develop the drone system for inspection at infrastructure equipment such as the bridge or tunnel.

\section{ACKNOWLEDGEMENTS}

This work was supported by JSPS KAKENHI Grant Number JP15K12598.

\section{REFERENCES}

[1] K. Nonami, F. Kendoul, S. Suzuki andW. Wang, D. Nakazawa, "Autonomous flying robots: unmanned aerial vehicles and micro aerial vehicles", Springer Japan, 2010.

[2] H. Takano andH. Toda, "Roll movement realized by yaw and roll command combination method of AR Drone 4 rotor helicopter for improving stability of automatic position stop", International Conference on Advanced Mechatronics (ICAM 2015), Waseda University, Tokyo, Japan, Dec.5-8, 2015, p.53.

[3] S. Azrad, F. Kendoul andK. Nonami, "Visual Servoing of Quadrotor Micro-Air Vehicle Using Color-Based Tracking Algorithm", Journal of System Design and Dynamics (JSME), Vol. 4, No. 2, 2010,pp. 255-268.

[4] T. K. Roy, M. Garratt, H. R. Pota andM. K. Samal, "Robust altitude control for a small helicopter by considering the ground effect compensation", Intelligent Control and Automation (WCICA), 10th World Congress, DOI:10.1109/WCICA.2012.6358168, 2010, pp. 1796-1800.

[5] A. C. Satici, H. Poonawala andM. W. Spong, "Robust Optimal Control of Quadrotor UAVs", IEEE Access, Vol. 1, DOI:10.1109/ACCESS.2013.2260794, 2013, pp. 79-93.

[6] J. Toledo, L. Acosta, M. Sigut, J. Felipe, "Stability Analisys of a Four Rotor Helicopter", Automation Congress, WAC '06. World, 2006, pp. 1-6.

[7] P. Castillo, A. Dzul, R. Lozano, "Real-time stabilization and tracking of a four-rotor mini rotorcraft", IEEE Transactions on Control Systems Technology, Vol.12,Issue: 4, DOI:10.1109/TCST.2004.825052, 2004, pp. 510-516.

[8] E. Altug, J. P. Ostrowski, C. J. Taylor, "Control of a quadrotor helicopter using dual camera visual feedback", International Journal of Robotics Research, Vol.24, No.5, 2005, pp. 329-341.

[9] B. Ludington, E. Johnson, G. Vachtservanos, "Augmenting UAV autonomy: vision-based navigation and target tracking for unmanned aerial vehicles", IEEE Robotics and Automation Magazine, Vol.13, No.3, 2006, pp. 63-71.

[10] H. Toda, H. Takano, "Effect of Discrete Yaw Direction Setting for 4 Roter Helicopter Control: Computer Simulation and AR. Drone Model Implementation", International Journal of Innovative Research in Advanced Engineering, Vol. 3, Issue 7, Paper ID JYAE10086, doi:10.17632/cj8f3v6csr.1, 2016.

[11] J. Engel, J. Sturm, D. Cremers, "Camera-based navigation of a low-cost quadrocopter", IEEE/RSJ International Conference on Intelligent Robots and Systems (IROS), DOI: 10.1109/IROS.2012.6385458, 2012.

[12] O. Andrisani, E. T. Kim, J. Schierman, F. P. Kuhl, "A nonlinear helicopter tracker using attitude measurements", IEEE Transactions on Aerospace and Electronic Systems, Vol. 27, Issue 1, DOI:10.1109/7.68146, 1991, pp. 40-47.

[13] L. Garcia-Delgado, A. Dzul, V. Santib, M. Llama, "Quad-rotors formation based on potential functions with obstacle avoidance", Control Theory and Applications, Vol. 6, Issue. 12, DOI:10.1049/iet-cta.2011.0370, 2012, pp. 1787-1802. 
[14] F. Kendoul, Z. Yu, K. Nonami, "Embedded autopilot for accurate waypoint navigation and trajectory tracking: Application to miniature rotorcraft UAVs", IEEE International Conference on Robotics and Automation, ICRA '09., DOI:10.1109/ROBOT.2009.5152549, 2009, pp. 2884-2890.

[15] E. Altu, J. P. Ostrowski, R. Mahony, "Control of a Quadrotor Helicopter Using Visual Feedback", Proceedings of the 2002 IEEE International Conference on Robotics and Automation, Washington DC, 2002, pp. 72-77.

[16] Y. Yoshihara, K. Watanabe, T. Iwatani, K. Hashimoto, "Image-based visual servo control for a micro helicopter under partial occlusions", Proceedings of 2007 JSME Conference on Robotics and Mechatronics, 2A2-A06, Akita, Japan, 2007.

[17] Ministry of Land (Japan), Liang maintenance technology site verification and evaluation of the results 2015, https://www.mlit.go.jp/road/ir/ir-council/pdf/yobo3_1_6.pdf (accessed on Aug. 2 2017)

[18] K. Nomura, "Industrial applications type electric multi-rotor helicopter, introduction of mini-surveyor and flight demonstration", Mini-surveyor consortium 2016, http://mec2.tm.chiba-u.jp/ nonami/consortium/outline.html/ (accessed on Aug.2 2017)

[19] (2016) Ministry of Land (Japan), "Results of on-site verification and evaluation of bridge maintenance technology", https://www.mlit.go.jp/common/001083014.pdf (accessed on Aug.2 2017)

[20] H. Toda, S. Honda, H. Takano, "Effectiveness of Fast Speed Yaw and Roll Control Switching Instead of Normal Roll Control for AR Drone 4 rotor Helicopter", International Journal of Innovative Research in Advanced Engineering, Vol. 3, Issue 7, Paper ID JYAE10081, doi:10.17632/y52bmbgtkg.1, 2016.

[21] S. Honda, H. Toda, M. Kitani, G. Capi, "Development of small size 4 rotor helicopter automatic position control algorithm", 32nd Annual Conference of the Robotics Society of Japan, 1D3-01, 2013.

[22] S. Honda, H. Toda, M. Kitani, G. Capi, “Development of 4 rotor automatic flight control system using image processing filters that can be self-position estimation in natural objects with the aim of the reactor building search", ROBOMECH2014, March, Toyama, 2014.

[23] K. Nonami, "Rotary Wing Aerial Robotics", Journal of Robotics Society of Japan, Vol. 24 (8), 2006, pp. 890-896.

[24] R. Mahony, T. Hamel, "Image-based visual servo control of aerial robotic systems using linear image features", IEEE Trans. on Robotics, Vol.21, No.2, 2005, pp. 227-239.

[25] K. Watanabe, Y. Iwatani, N. Kenichiro, K. Hashimoto, “A vision-based support system for micro helicopter control”, Proceedings of Conference on Robotics and Mechatronics, 1P1-F13, Nagano, Japan, June 5-7, 2008.

[26] Y. Kubota, T. Iwatani, "Dependable visual servoing of a small-scale helicopter with a wireless camera", Proceedings of Conference on Robotics and Mechatronics, 1A2-O15, Okayama, Japan, May 26-28, 2011.

[27] O. Meister, N. Frietsch, C. Ascher, G. F. Trommer, "Adaptive path planning for a VTOL-UAV", IEEE International Coference on Position, Location and Navigation Symposium, DOI:10.1109/PLANS.2008.4570046, 2008, pp. 1252-1259.

[28] S. Yoshida, "Engneering navi”, library of ARDroneForP5, http://kougaku-navi.net/ARDroneForP5/ (accessed on Aug.2 2017). 